> L'intérêt actuel de la communauté médicale pour les anticorps monoclonaux doit beaucoup au succès clinique d'un anticorps anti-CD20, le rituximab (MabThera ${ }^{\circledR}$ ). Au cours des dix années qui viennent de s'écouler, cet anticorps a permis de démontrer la pertinence du concept de thérapie ciblée et s'est imposé dans le traitement des pathologies lymphoïdes B. Dans le même temps, grâce à une meilleure compréhension des mécanismes d'action opérant in vivo, il a permis l'avènement des anticorps de demain doués d'une plus grande capacité de recrutement des effecteurs cellulaires immuns. Dans cette mise au point sur l'expérience clinique en oncohématologie des anticorps monoclonaux, nous décrirons les résultats obtenus avec ceux dont l'utilisation a été autorisée et discuterons les enseignements de dix ans d'utilisation de cette nouvelle classe médicamenteuse. <

\section{Anticorps \\ monoclonaux \\ thérapeutiques en \\ oncohématologie}

Guillaume Cartron, Jean-François Rossi

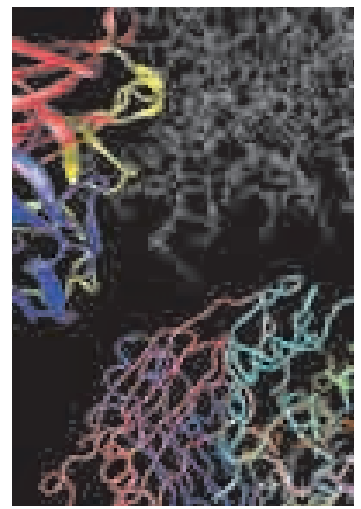

comme le traitement de première intention des LDGCB. Une amélioration similaire de la survie a été également observée dans les essais cliniques évaluant l'administration de cette association en première ligne dans les lymphomes folliculaires (LF) [2-5]. L'adjonction du rituximab a ainsi permis un gain significatif de survie de l'ordre de $10 \%$ à quatre ans. C'est aussi dans cette indication qu'est réapparu le concept de traitement d'entretien initialement développé au début des années 1970 avec la chimiothérapie, puis délaissé dans cette indication en raison de son manque d'efficacité. Ainsi, cet anticorps administré tous les deux ou trois mois pendant deux ans après une phase d'induction associant rituximab et chimiothérapie a permis d'améliorer la survie des patients atteints d'un LF en rechute [6]. L'intérêt du rituximab a aussi été montré dans les lymphomes à cellules du manteau ${ }^{2}[7,28]$, mais son bénéfice thérapeutique n'est pas établi dans les autres types histologiques de lymphome B. Dans la leucémie lymphoïde chronique (LLC), les réponses complètes sont rares mais le taux de réponse ainsi que la survie sans progression sont augmentés significativement lorsque le rituximab est ajouté au traitement conventionnel associant fludarabine et

\footnotetext{
${ }^{2}$ Les lymphomes du manteau sont «des formes hétérogènes de lymphomes non hodgkiniens impliquant une prolifération lymphoïde B dont l'origine est la zone du manteau des follicules lymphoïdes associés aux muqueuses » (reproduit de [28]).
}

${ }^{1}$ La molécule CD20 est une protéine transmembranaire non glycosylée présente à la surface des lymphocytes B matures et de leurs précurseurs. Sa fonction précise est inconnue, mais elle pourrait être impliquée dans la signalisation, ce que suggère la présence d'un domaine intracellulaire riche en séquences de phosphorylation, et qui peut s'associer avec des kinases de la famille Src.

\section{Indications cliniques actuelles} $45 \%$ pour les patients recevant uniquement une chimi thérapie [1]. Aujourd'hui cette association est reconnue 
cyclophosphamide en première ligne ou en rechute. Il est vraisemblable, comme le suggère l'étude de phase I, que la dose retenue dans cette indication $\left(500 \mathrm{mg} / \mathrm{m}^{2}\right)$ ne soit pas optimale [8]. Un essai de phase II semble confirmer un taux de réponse supérieur lorsque des doses supérieures de rituximab sont administrées [9]. Récemment, l'association du rituximab à la fludarabine et le cyclophosphamide ont montré une augmentation significative de la survie sans progression chez des patients atteints de LLC en rechute ou en première ligne de traitement.

L'ibritumomab-tiuxétan (mu-IgGl, Zévalin ${ }^{\circledR}$ ) est un anticorps anti-CD20 couplé à l'yttrium ${ }^{90}$ ( $\beta$-émetteur). Le potentiel thérapeutique de la radio-immunothérapie est évident pour ces tumeurs réputées radiosensibles $(\rightarrow)$. L'autre intérêt est que l'administration de l'ibritumomab-tiuxétan ne nécessite pas de mesure de radioprotection et peut donc être réalisée en ambulatoire. Environ $80 \%$ des patients $(\rightarrow)$ voir A. Pèlegrin et $B$. Robert, page 1155 ; J. Barbet et al., page 1039 atteints d'un lymphome indolent présentent une réponse clinique après une seule dose, et chez $25 \%$ environ des patients cette réponse se prolonge au-delà d'un an. L'administration de cet anticorps chez des patients « réfractaires » au rituximab a permis d'obtenir une rémission complète chez $15 \%$ d'entre eux [10]. De même, son administration à des patients en rechute d'un lymphome indolent augmente significativement le taux de réponse comparativement au rituximab [11]. Une étude plus récente a montré l'intérêt d'une injection d'ibritumomab-tiuxétan en consolidation d'un traitement chimiothérapique chez des patients atteints d'un LF [12]. Cependant cet intérêt reste à confirmer chez les patients recevant au préalable une association rituximab-chimiothérapie. Plus récemment, l'adjonction d'ibritumomab-tiuxétan à la chimiothérapie de conditionnement administrée avant une autogreffe de cellulessoucheshématopoiétiques a étéproposée. Sonbénéficethérapeutique dans cette indication doit être évalué par des études contrôlées.

L'lode $^{131}$ tositumomab (mu-lgG2a, Bexxar ${ }^{\circledR}$ ) n'est pas commercialisé en Europe. II s'agit d'un émetteur $\gamma$ et son utilisation requiert un isolement du patient en chambre protégée. En monothérapie, cet anticorps a permis d'obtenir une rémission complète chez près de $75 \%$ des patients atteints d'un LF et $59 \%$ de survie sans progression à cinq ans [13]. De même l'administration du $1^{131}$ tositumomab a permis l'obtention de survies prolongées sans progression lorsqu'il est administré après une phase de chimiothérapie [14]. L'évaluation de cet anticorps souffre cependant de l'absence d'études contrôlées.

\section{Anticorps anti-CD52}

L'alemtuzumab (hu-lgGl, MabCampath ${ }^{\circledR}$ ) est un anticorps anti-CD52 ${ }^{3}$ indiqué chez les patients atteints d'une LLC en rechute. Cet anticorps est habituellement utilisé seul dans cette indication. Environ $30 \%$ des patients rechutant après un traitement par fludarabine répondent à ce traitement [15]. Ils sont près de $80 \%$ lorsque I'on utilise cet anticorps en première ligne [16]. L'expérience clinique a montré qu'il était moins efficace chez les patients présentant un syndrome

${ }^{3}$ L'antigène CD52, une glycoprotéine ancrée à la membrane par un résidu glycophosphatiodylinositol, est exprimé par les lymphocytes B et T, par les monocytes et les cellules natural killer. Sa fonction est pour l'instant inconnue. tumoral important (tumeur supérieure à $5 \mathrm{~cm}$ ). Son utilisation clinique est aussi limitée par les réactions (frissons, fièvre, hypotension, etc.) observées lors de l'administration intraveineuse. La voie sous-cutanée améliore significativement la tolérance et constitue aujourd'hui la voie d'administration la plus utilisée. Cependant, l'incidence accrue des infections opportunistes à cytomegalovirus et à herpes simplex constitue aujourd'hui la limite majeure de son $(\rightarrow)$ voir $\varepsilon$. Rigal et utilisation $(\rightarrow)$.

al. page 1135

\section{Anticorps anti-CD33}

Le gemtuzumab ozogamycine (hu-IgG4, Mylotarg ${ }^{\circledR}$ ) est un anticorps humanisé couplé à la calichéamycine ${ }^{4}$ utilisé aux États-Unis chez les patients âgés de plus de soixante ans en rechute d'une leucémie aiguë myéloïde. Dans cette indication, il permet d'obtenir environ $30 \%$ de réponse objective dont environ $12 \%$ de réponses complètes. Une étude plus récente [17] a montré que son association à une chimiothérapie en première ligne permettait d'obtenir une réduction significative du nombre de rechutes. Cependant, cette étude n'a pas permis de mettre en évidence une amélioration de la survie des patients. Le développement de cette molécule pourrait être limité par la survenue d'une toxicité hépatique significative pouvant conduire à la survenue d'une véritable maladie occlusive ${ }^{5}$. Cette toxicité conduit à recommander un délai d'au moins quatre mois entre l'utilisation de cet $\mathrm{Acm}$ et la réalisation d'une greffe allogénique, cette dernière constituant elle-même un facteur de risque d'hépatotoxicité.

\section{Les enseignements de dix ans d'utilisation des Acm en oncologie hématologique}

Le développement de cette nouvelle classe thérapeutique a permis de retenir plusieurs enseignements utiles à la conception d'une nouvelle génération d'anticorps thérapeutiques.

\section{Enseignements cliniques}

Malgré leur efficacité démontrée, ces Acm, lorsqu'ils sont utilisés seuls, induisent de faibles taux de rémission complète, et c'est l'association à la chimiothérapie qui a permis leur développement. En effet, et contrairement aux espoirs suscités par ces thérapeutiques ciblées, les Acm

${ }^{4}$ La diméthyl hydrazide $\mathrm{N}$-acétyl-calichéamycine, une anthracycline cytotoxique, est responsable des effets biologiques du conjugué.

${ }^{5}$ «Atteinte des petites veines hépatiques qui réalise une obstruction non thrombotique, par épaississement sous endothélial, fait d'œdème puis de fibrose lâche. Les manifestations sont celles d'un syndrome de Budd-Chiari. La gravité est variable. L'étiologie est toxique, prise de dérivés de pyrolizidine, ou secondaire à une irradiation corporelle ou à l'administration d'antinéoplasiques. » (adapté de Orphanet). 
n'ont pas, ou à de rares exceptions près, permis d'éviter l'usage d'agents cytotoxiques. Néanmoins, l'arrivée de ces molécules a réduit de manière significative les indications d'intensification thérapeutique avec greffe de cellules souches hématopoïétiques autologues comme traitement adjuvant $^{6}$. Ainsi, l'indication de cette procédure n'est plus d'actualité dans les LF non préalablement traités et une étude randomisée évalue actuellement son intérêt comparativement à l'association R-CHOP dans les LDGCB.

Il est intéressant de noter que les modalités pratiques d'administration (dose, schéma) des Acm non radiomarqués reposent largement sur l'empirisme. Le développement clinique du rituximab en est un parfait exemple. Ainsi les phases I testant le rituximab [18, 19] n'ontelles pas permis de définir de dose limitante toxique, un résultat sur lequel on se fonde pour définir la dose à administrer lors des phases II et tester l'efficacité du médicament dans différentes pathologies $(\rightarrow)$. De même, aucune rela-

$\rightarrow$ voir G. Paintaud, page 1057

tion dose-effet n'a été très clairement mise en

évidence. Ainsi, le choix de la dose utilisée dans la phase II (375 mg/ $\mathrm{m}^{2}$ ) a reposé essentiellement sur des considérations industrielles (disponibilité et coût du produit). De la même manière, le choix du nombre de perfusions (quatre) et de l'intervalle entre chaque administration (une semaine) ne repose pas sur des bases scientifiques solides. Aujourd'hui, c'est finalement son association à la chimiothérapie qui, pour des raisons pratiques, dicte le rythme d'administration toutes les trois semaines et le nombre de cycles (six à huit).

Le développement rapide des indications des Acm doit beaucoup à la bonne tolérance de ces molécules. II faut cependant nuancer cette affirmation car la tolérance dépend clairement de la cible. Par exemple, la stratégie anti-CD20 s'accompagne d'un très bon index thérapeutique

${ }^{6}$ L'administration de cellules souches hématopoïétiques autologues permet d'utiliser des doses plus importantes de chimiothérapie, car elle en atténue la toxicité hématologique en raccourcissant la période d'aplasie et en en diminuant l'intensité.
(Tableau I) et les effets indésirables sont observés essentiellement lors de la première perfusion (frisson, fièvre, hypotension). En revanche, l'utilisation d'Acm anti-CD52 peut se compliquer de réactivations virales (cytomegalovirus) et d'infections opportunistes $(\rightarrow)$ voir $\boldsymbol{\varepsilon}$. Rigal et $(\rightarrow)$. Concernant la radio-immuno- al., page 1135 thérapie, sa tolérance est acceptable (cytopénies retardées) pour peu que l'on écarte les patients ayant un infiltrat lymphomateux médullaire. De manière plus générale, il faut rappeler que nous avons un recul encore trop modeste pour apprécier réellement les effets à long terme de ce type de traitement $(\rightarrow)$.

$(\rightarrow)$ voir J. Barbet et al., page 1039

\section{Enseignements biologiques}

Le développement important de cette classe de médicaments depuis dix ans (Tableau II) a suscité beaucoup d'interrogations quant à leurs mécanismes d'action in vivo. Là encore c'est le modèle du rituximab et du lymphome B qui a fait progresser les connaissances. Ainsi, si les mécanismes d'action du rituximab in vitro sont parfaitement décrits (apoptose, lyse dépendante du complément et cytotoxicité médiée par les récepteurs de la portion Fc: FcyR) [20], leur survenue in vivo et leur part relative dans l'effet thérapeutique restent méconnues. Cette question a rapidement été soulevée au vu de la variabilité de la réponse thérapeutique: ainsi un faible nombre de patients est en rémission complète à l'issue d'un traitement par rituximab alors que la plupart présentent une réponse partielle à l'anticorps. Ainsi est-il apparu qu'une meilleure compréhension des mécanismes déterminant cette variabilité de la réponse à l'anticorps

\begin{tabular}{|c|c|c|}
\hline $\begin{array}{c}\text { Rituximab } \\
\left.\text { (MabThéra }{ }^{\circledR}\right)\end{array}$ & $\begin{array}{l}\text { Ibritumomab-tiuxétan } \\
\qquad\left(\text { Zévalin }{ }^{\circledR}\right)\end{array}$ & $\begin{array}{l}\text { Gemtuzumab ozogamycine } \\
\left.\text { (Mylotarg }{ }^{\circledR}\right)\end{array}$ \\
\hline $\begin{array}{l}\text { Réaction à la première perfusion (frissons, fiè- } \\
\text { vre, tremblements) chez } 50 \% \text { des patients avec } \\
\text { dans de rares cas un syndrome de relargage } \\
\text { cytokinique (hypotension, bronchospasme) }\end{array}$ & $\begin{array}{l}\text { Myélosuppression retardée } 4 \text { à } 6 \text { semaines après } \\
\text { l'administration }\end{array}$ & $\begin{array}{l}\text { Réaction à la première perfusion (fris- } \\
\text { sons, fièvre, tremblements) }\end{array}$ \\
\hline Lymphopénie B & Myélodysplasie et leucémie aiguë secondaires & Myélosuppression \\
\hline $\begin{array}{l}\text { Neutropénie tardive (> } 12 \text { semaines après la fin } \\
\text { du traitement) }\end{array}$ & Lymphopénie B & $\begin{array}{l}\text { Hépatite mixte pouvant évoluer vers } \\
\text { une maladie veino-occlusive }\end{array}$ \\
\hline Réduction des IgG & Apparition d'HAMA & \\
\hline \multicolumn{3}{|l|}{ Réactivation hépatite $B$ ancienne } \\
\hline \multicolumn{3}{|l|}{ Leucoencéphalite multifocale progressive } \\
\hline $\begin{array}{l}\text { Majoration ( } \times 2 \text { ) du risque d'infection en cas de } \\
\text { traitement d'entretien }\end{array}$ & & \\
\hline
\end{tabular}

Tableau I. Principaux effets indésirables des anticorps monoclonaux utilisés en oncohématologie. HAMA : human anti-mouse antibodies. 


\begin{tabular}{|c|c|c|c|}
\hline & Cible & Pathologie & $\begin{array}{c}\text { Développement } \\
\text { clinique }\end{array}$ \\
\hline \multicolumn{4}{|l|}{ Anticorps « nus 》 } \\
\hline Zanolimumab & CD4 & Lymphome T & Phase II \\
\hline $\mathrm{XmAb} 5574$ & CD19 & Lymphome/leucémie & Phase I \\
\hline Veltuzumab & CD20 & Lymphome B & Phase II \\
\hline Ofatumumab & CD20 & Lymphome B/LLC & Phase III \\
\hline GAl01 & CD20 & Lymphome B/LLC & Phase I/II \\
\hline EMAB-6 & CD20 & Lymphome B/LLC & Phase I \\
\hline PR0131921 & CD20 & Lymphome B/LLC & Phase I \\
\hline AME133 & CD20 & Lymphome B/LLC & Phase I \\
\hline KM3065 & CD20 & Lymphome B/LLC & Phase I \\
\hline Épratuzumab & CD22 & Lymphome B & Phase II \\
\hline CMC544 & CD22 & Lymphome B & Phase II \\
\hline Lumiliximab & CD23 & LLC & Phase III \\
\hline SGN30 & CD30 & Hodgkin/Lymphome T & Phase I/II \\
\hline MDX060 & CD30 & Hodgkin/Lymphome T & Phase I/II \\
\hline HUM195 & CD33 & Leucémie aiguë & Phase III \\
\hline SGN70 & CD70 & Lymphome & Phase I \\
\hline Mylatuzumab & CD74 & Lymphome & Phase I \\
\hline Galiximab & CD80 & Lymphome B & Phase III \\
\hline Apolizumab & $\mathrm{DR}$ & Lymphome B/LLC & Phase II \\
\hline HGS-ETRl & TRAIL-Rl & Lymphome & Phase II \\
\hline \multicolumn{4}{|l|}{ Anticorps 《 couplés » } \\
\hline${ }^{131} \mid-$ Lym l & DR10 & Lymphome B/LLC & Phase II \\
\hline${ }^{90} y-C D 19$ & CD19 & Leucémie & Phase II \\
\hline${ }^{90} y-L L 2$ & CD22 & Lymphome B/LLC & Phase II \\
\hline Inotuzumab-ozogamycine & CD22 & Lymphome & Phase II \\
\hline${ }^{131} \mid-M B 1$ & $\mathrm{CD} 37$ & Lymphome & Phase I \\
\hline${ }^{131} \mid-B C 8$ & CD45 & Leucémie/greffe & Phase II \\
\hline${ }^{188}$ Re-anti-CD 66 & CD66 & Leucémie/greffe & Phase II \\
\hline
\end{tabular}

influence l'affinité de ce récepteur du fragment Fc pour sa cible. Ainsi les patients homozygotes pour l'allotype de meilleure affinité (V) ont une meilleure réponse au rituximab que les patients porteurs de l'allotype de plus faible affinité (F). Cette découverte a permis de souligner l'importance de l'interaction Fc-FcyRIIla, un élément à prendre en compte pour le développement d'Acm de nouvelle génération

$(\rightarrow)$. Aujourd'hui, c'est la

$(\rightarrow)$ voir R. Abès stratégie de l'afucosylation

et al., page 1011

de l'oligosacharide situé sur l'asparagine 297 qui apparaît la plus avancée pour optimiser la fonction effectrice ADCC induite par l'anticorps $\rightarrow$. L'étude de phase I testant le premier anticorps

$(\rightarrow)$ voir A. Beck et al., page 1024 anti-CD20 afucosylé (GA101, Roche) vient d'être publiée et elle indique des résultats prometteurs pour cette nouvelle génération d'anticorps [22]. II fait peu de doutes que cette stratégie sera très prochainement adaptée aux autres anticorps dont l'activité thérapeutique semble aussi influencée par le polymorphisme FcyRIIla-158VF (trastuzumab, cétuximab) [23, 24].

L'importance des mécanismes relayés par le récepteur Fc $\gamma R$ du fragment Fc permet aussi de souligner les difficultés liées à l'utilisation des modèles animaux dans l'expertise préclinique (toxicologique) ou expérimentale (mécanisme d'action). En effet, il apparaît de plus en plus évident que l'éloignement génétique des animaux utilisés pour l'expérimentation (modèles murins, cynomolgus) appelle à la prudence dans l'extrapolation des résultats obtenus en clinique humaine $[25,26]$.

\section{Conclusion et perspectives}

Les anticorps monoclonaux ont permis ces dernières années des progrès thérapeutiques considérables dans le traitement des hémopathies lymphoïdes B. L'enjeu aujourd'hui est d'optimiser leur utilisation pour les rendre plus efficaces. Cela passe notamment par une meilleure connaissance de leurs mécanismes d'action et par une utilisation pourrait permettre une optimisation de l'effet thérapeutique. Une avancée significative dans ce domaine a été apportée par la démonstration de l'influence du polymorphisme du récepteur FcyRIIla-158VF [21] exprimé par les cellules natural killer (NK) et les monocytes. En effet, ce polymorphisme plus rationnelle. Des anticorps améliorant I'ADCC vont être prochainement disponibles mais d'autres stratégies d'optimisation, comme l'utilisation d'adjuvants par exemple sous forme de cytokines (granulocyte- 
macrophage colony-stimulating factor ou GM-CSF, interféron, interleukine-2, etc.) [27] sont aussi possibles et doivent être évaluées. La limite principale de cette approche est la définition du bon antigène cible qui seule permettra d'assurer à un Acm son efficacité et sa bonne tolérance. La place aujourd'hui occupée par le rituximab démontre la pertinence de la stratégie anti-CD20 mais ne doit pas empêcher de relever le défi de la recherche de nouvelles cibles thérapeutiques. $\diamond$

\section{SUMMARY}

\section{Therapeutic monoclonal antibodies in onco-hematology}

Rituximab, a chimeric monoclonal anti-CD20 antibody, was introduced into clinical practice in 1997, and has proven to be highly effective in the treatment of B-lymphoproliferative disorders and autoimmune diseases. Despite such success, in vivo mechanisms of action of anti-CD20 have only recently began to be unraveled, pointing to the crucial role of antibody-dependent cellular cytotoxicity response mediated through Fc $\gamma$ receptor signalling. Better understanding of pharmacokinetics and factors influencing individual exposure mediated through anti-CD20 will allow to engineer these molecules to increase their effector responses. Meanwhile, other formats have also been investigated, such as radiolabeled anti-CD20, or coupling of antibodies to cytotoxic drugs such as anti-CD33 used in myeloid leukemia. However these antibodies are used in combination with standard chemotherapy and cannot substitute for cytotoxic drugs. This review summarizes the knowledge acquired through our clinical use of anti-CD20 and authorized monoclonal antibodies in oncohematology and proposes some news areas that will lead to the development of new and more effective therapeutic strategies. $\diamond$

\section{CONFLIT D'INTÉRÊTS}

Les auteurs déclarent n'avoir aucun conflit d'intérêts concernant les données publiées dans cet article.

\section{RÉFÉRENCES}

1. Feugier P, Van Hoof A, Sebban C, et al. Long-term results of the R-CHOP study in the treatment of elderly patients with diffuse large B-cell lymphoma : a study by the Groupe d'étude des lymphomes de l'adulte. J Clin Oncol $2005 ; 23$ : 4117-26.

2. Hiddemann W, Kneba M, Dreyling $M$, et al. Frontline therapy with rituximab added to the combination of cyclophosphamide, doxorubicin, vincristine, and prednisone (CHOP) significantly improves the outcome for patients with advanced-stage follicular lymphoma compared with therapy with CHOP alone : results of a prospective randomized study of the german low-grade lymphoma study group. Blood $2005 ; 106: 3725-32$

3. Herold M, Haas A, Srock S, et al. Rituximab added to first-line mitoxantrone, chlorambucil, and prednisolone chemotherapy followed by interferon maintenance prolongs survival in patients with advanced follicular lymphoma : an East German study group hematology and oncology Study. J Clin Oncol $2007 ; 25$ : 1986-92.

4. Marcus RE, Solal-Celigny P, Imrie K, et al. MabThera (Rituximab) plus cyclophosphamide, vincristine and prednisone (R-CVP) chemotherapy improves survival in previously untreated patients with advanced follicular non-Hodgkin's lymphoma (NHL). Blood 2006 ; 108: 481a.

5. Salles G, Mounier N, de Guibert S, et al. Rituximab combined with chemotherapy and interferon in follicular lymphoma patients : results of the GELA-GOELAMS FL2000 study. Blood 2008 ; 112 : 4824-31.

6. Van Oers MH, Klasa R, Marcus RE, et al. Rituximab maintenance improves clinical outcome of relapsed/resistant follicular non-Hodgkin lymphoma in patients both with and without rituximab during induction : results of a prospective randomized phase 3 intergroup trial. Blood 2006; $108: 3295-301$.

7. Forstpointner R, Unterhalt M, Dreyling M, et al. Maintenance therapy with rituximab leads to a significant prolongation of response duration after salvage therapy with a combination of rituximab, fludarabine, cyclophosphamide, and mitoxantrone (R-FCM) in patients with recurring and refractory follicular and mantle cell lymphomas : results of a prospective randomized study of the German low grade lymphoma study group (GLSG). Blood $2006 ; 108$ : 4003-8.
8. O'Brien SM, Kantarjian $\mathrm{H}$, Thomas DA, et al. Rituximab dose-escalation trial in chronic lymphocytic leukemia. J Clin Oncol 2001 ; 19 : 2165-70.

9. James DF, Castro JE, Sandoval-Sus JD, et al. Rituximab and high-dose methylprednisolone for the initial treatment of chronic lymphocytic leukemia is associated with promising clinical activity and minimal hematologic toxicity. Blood 2008; 112 : 47.

10. Witzig TE, Flinn IW, Gordon LI, et al. Treatment with ibritumomab tiuxetan radioimmunotherapy in patients with rituximab-refractory follicular nonHodgkin's lymphoma. J Clin Oncol $2002 ; 20$ : 3262-9.

11. Witzig TE, Gordon LI, Cabanillas F, et al. Randomized controlled trial of yttrium90-labeled ibritumomab tiuxetan radioimmunotherapy versus rituximab immunotherapy for patients with relapsed or refractory low-grade, follicular, or transformed B-cell non-Hodgkin's lymphoma. J Clin Oncol 2002 ; 20 : 2453-63.

12. Morschhauser F, Radford J, Van Hoof A, et al. Phase III trial of consolidation therapy with yttrium-90-ibritumomab tiuxetan compared with no additional therapy after first remission in advanced follicular lymphoma. J Clin Oncol $2008 ; 26: 5156-64$.

13. Kaminski MS, Tuck M, Estes J, et al. 1311-tositumomab therapy as initial treatment for follicular lymphoma. N Engl J Med 2005 ; 352 : 441-9.

14. Press OW, Unger JM, Braziel RM, et al. Phase II trial of CHOP chemotherapy followed by tositumomab/iodine I-131 tositumomab for previously untreated follicular non-Hodgkin's lymphoma : five-year follow-up of Southwest oncology group protocol S9911.J Clin Oncol 2006 ; 24 : 4143-9.

15. Keating MJ, Flinn I, Jain V, et al. Therapeutic role of alemtuzumab (Campath$1 \mathrm{H})$ in patients who have failed fludarabine : results of a large international study. Blood 2002; 99 : 3554-61.

16. Hillmen P, Skotnicki $A B$, Robak $T$, et al. Alemtuzumab compared with chlorambucil as first-line therapy for chronic lymphocytic leukemia.J Clin Oncol $2007 ; 25: 5616-23$.

17. Burnett AK, Kell WJ. The addition of gemtuzumab ozogamycin toinduction therapy for AML improves disease free survival without extra toxicity. Blood $2006 ; 108: 13$.

18. Maloney DG, Grillo-Lopez AJ, Bodkin DJ, et al. IDEC-C2B8 : results of a phase multiple-dose trial in patients with relapsed non-Hodgkin's lymphoma.J Clin Oncol 1997; 15: 3266-74.

19. Maloney DG, Liles TM, Czerwinski DK, et al. Phase I clinical trial using escalating single-dose infusion of chimeric anti-CD20 monoclonal antibody (IDEC-C2B8) in patients with recurrent B-cell lymphoma. Blood $1994 ; 84: 2457-66$.

20. Cartron G, Watier H, Golay J, Solal-Celigny P. From the bench to the bedside: ways to improve rituximab efficacy. Blood $2004 ; 104$ : 2635-42.

21. Cartron G, Dacheux L, Salles G, et al. Therapeutic activity of humanized antiCD20 monoclonal antibody and polymorphism in IgG Fc receptor FcgRIIla gene. Blood $2002 ; 98: 754-8$.

22. Salles G, Morschhauser F, Cartron G, et al. A Phase I/II study of R05072759 (GA101) in patients with relapsed/refractory $\mathrm{CD}_{20} \mathrm{O}^{+}$malignant disease. Blood $2008 ; 112: 234$.

23. Bibeau F, Crapez $\varepsilon$, Di Fiore F, et al. Association of FcgRIla and FcgRIla polymorphisms with clinical outcome in metastatic colorectal cancer patients (mCRC) treated with cetuximab and irinotecan. J Clin Oncol 2008; 26: 11004.

24. Musolino A, Naldi N, Bortesi B, et al. Immunoglobulin $G$ fragment $C$ receptor polymorphisms and clinical efficacy of Trastuzumab-based therapy in patients With HER-2/neu-positive metastatic breast cancer. J Clin Oncol 2008 ; 26 : 1789-96.

25. Nimmerjahn F, Ravetch JV. Fcgamma receptors as regulators of immune responses. Nat Rev Immunol $2008 ; 8$ : 34-47.

26. Loisel $S$, Ohresser M, Pallardy M, et al. Relevance, advantages and limitations of animal models used in the development of monoclonal antibodies for cancer treatment. Crit Rev Oncol Hematol 2007 ; 62 : 34-42.

27. Cartron G, Zhao-Yang L, Baudard M, et al. Granulocyte-macrophage colonystimulating factor potentiates rituximab in patients with relapsed follicular lymphoma: results of a phase II study. J Clin Oncol 2008; 26:2725-31.

28. Lobry C, Weil R. New Bcllo regulation mechanisms : a step in the comprehension of what has occurred in MALT lymphomas? Med Sci (Paris) 2007 ; 23 : 353-5.
TIRÉS À PART

G. Cartron 